\title{
Efeito da classificação dos ovos sobre a uniformidade, o desempenho e o rendimento de abate de frangos de corte
}

\author{
[Effect of egg classification prior to setting on broiler chicken uniformity, performance and meat yields] \\ J.S.R. Rocha ${ }^{1}$, L.J.C. Lara $^{2}$, N.C. Baião ${ }^{2 *}$, S.V. Cançado ${ }^{2}$, M.V. Triginelli ${ }^{3}$, J.F.C. Leite ${ }^{3}$ \\ ${ }^{1}$ Aluna de pós-graduação - EV-UFMG - Belo Horizonte, MG \\ ${ }^{2}$ Escola de Veterinária - UFMG \\ Caixa Postal 567 \\ 30123-970 - Belo Horizonte, MG \\ ${ }^{3}$ Aluno de graduação - EV-UFMG - Belo Horizonte, MG
}

\begin{abstract}
RESUMO
Avaliou-se a influência da classificação dos ovos no incubatório sobre a uniformidade, o desempenho e o rendimento de abate dos frangos de corte, utilizando-se 140 machos e 140 fêmeas nascidos de cada um dos seguintes tratamentos: A - ovos com pesos entre 66 e $72 \mathrm{~g}, 33,3 \%$ deles originados de matrizes com diferentes idades; $\mathrm{B}$ - ovos com pesos entre 58 e $65 \mathrm{~g}$, 33,3\% deles originados de matrizes com diferentes idades; C - ovos com pesos entre 52 e 57g, 33,3\% deles originados de matrizes com diferentes idades; D - ovos com pesos entre 52 e 72g, produzidos por matrizes com 31 semanas; $\mathrm{E}$ - ovos com pesos entre 52 e $72 \mathrm{~g}$, produzidos por matrizes com 38 semanas; e F - ovos com pesos entre 52 e $72 \mathrm{~g}$, produzidos por matrizes com 43 semanas. $\mathrm{O}$ delineamento experimental foi inteiramente ao acaso, em esquema fatorial 6 x 2 (seis categorias de peso dos ovos x dois sexos), com quatro repetições de 35 aves. O período de criação dos frangos foi de um a 44 dias de idade, e o abate foi feito aos 45 dias. A classificação dos ovos nos tratamentos A, B e C, visando limitar as categorias de peso dos ovos, não foi eficaz para manter a melhor uniformidade dos frangos com 44 dias de idade. $\mathrm{O}$ peso do frango aos 44 dias está positivamente correlacionado ao peso do pinto aos sete dias, exceto em frangos provenientes do tratamento D. $\mathrm{O}$ desempenho dos frangos e o rendimento de abate não foram influenciados pela classificação dos ovos no incubatório.
\end{abstract}

Palavras-chave: frango de corte, classificação dos ovos, incubação, uniformidade, desempenho

\begin{abstract}
The effect of egg classification on uniformity, performance, and meat yield of broiler chicks was evaluated using 140 males and 140 females hatched from one of each of the following treatments: A eggs weighting from 66 to $72 \mathrm{~g}, 33,3 \%$ of them produced by broiler breeders of different ages; $B$ - eggs weighting from 58 to $65 \mathrm{~g}, 33,3 \%$ of them produced by broiler breeders of different ages; $C$ - eggs weighting from 52 to $57 \mathrm{~g}, 33,3 \%$ of them produced by broiler breeders of different ages; $D$-eggs from 31-wk-old broiler breeders, weighting from 52 to 72g; E - eggs from 38-wk-old broiler breeders, weighting from 52 to $72 \mathrm{~g}$; and $F$ - eggs from 43-wk-old broiler breeders, weighting from 52 to $72 \mathrm{~g}$. It was used a completely randomized design, in a $6 \times 2$ factorial (six categories of egg classification $x$ two genders) with four replicates of 35 bird each. The chicks were raised from one to 44-day-old and slaughtered at 45-day-old. Sorting eggs by weight prior to incubation was not efficient to keep the better broiler chickens uniformity until they were 44-day-old. A significant and positive relationship between 7day-old chick weight and broiler weight at slaughtering age was observed, except in chicks from treatment D. Broiler performance and meat yield were not affected by egg classification.
\end{abstract}

Keywords: broiler chicken, egg classification, incubation, uniformity, performance

Recebido em 2 de abril de 2007

Aceito em 12 de agosto de 2008

*Autor para correspondência (corresponding author)

E-mail: baiao@vet.ufmg.br

Apoio: $\mathrm{CNPq} / \mathrm{FAPEMIG}$ 


\section{INTRODUÇÃO}

O efeito do peso do ovo sobre o peso do frango ao abate não está bem elucidado e parece ser afetado por fatores como linhagem e idade das reprodutoras. Sinclair et al. (1990) observaram alta correlação entre os pesos do ovo e do pinto à eclosão, porém, à medida que as aves cresceram até seis semanas de idade, o valor dessas estimativas diminuiu consideravelmente. Pinchasov (1991), ao avaliar o peso de pintos descendentes de matrizes com 52, 55 e 57 semanas de idade, concluiu que a vantagem do maior peso inicial dos nascidos de ovos mais pesados diminuiu rapidamente após a eclosão e que o principal fator que afetou o peso final foi o consumo de ração.

Segundo Wilson (1991), há um consenso geral na literatura de que pintos mais pesados ao nascimento resultam em frangos mais pesados ao abate. De acordo com Baião (2000) e Tona et al. (2005), o peso do frango ao abate é diretamente proporcional ao peso do pinto aos sete dias de idade. A correlação estimada foi alta e positiva ( $\mathrm{r}$ $=0,6$ a 0,9$)$ entre os pesos nessas idades.

Tona et al. (2004) pesaram individualmente os pintos produzidos por matrizes Cobb com $35 \mathrm{e}$ 45 semanas de idade quando estes estavam com um, sete, 14, 21, 28, 35 e 42 dias de idade. Esses autores encontraram significativa correlação entre o peso do frango aos 42 dias com o peso do pinto em todas as idades, exceto com um dia. Os pintos de matrizes com 35 semanas de idade foram mais leves até 14 dias de idade que os pintos originados de matrizes com 45 semanas. No entanto, após a terceira semana de idade, os pintos descendentes de reprodutoras mais jovens apresentaram alta taxa de crescimento, atingindo, aos 42 dias de idade, pesos semelhantes aos dos descendentes de matrizes mais velhas.

Ao compararem o desempenho de progênies de matrizes Ross com 32 e 41 semanas de idade, Joseph e Moran Jr. (2005) observaram que a diferença entre os pesos dos pintos à eclosão e aos oito dias de idade desapareceu aos 16 dias de idade. Às seis semanas de idade, os frangos descendentes das duas idades das matrizes apresentaram peso corporal semelhante. Não houve diferença significativa entre as taxas de mortalidade observadas nas duas progênies, e a conversão alimentar dos frangos descendentes de matrizes com 32 semanas foi melhor que a dos originados de matrizes com 41 semanas.

$\mathrm{Na}$ avicultura industrial, existe um conceito de que, para se obter lotes de frangos de corte com boa uniformidade de peso, estes têm que ser originados de ovos com pesos semelhantes. Baseado neste aspecto, muitos incubatórios utilizam o peso dos ovos como único critério de classificação para a obtenção de grande quantidade de pintos "uniformes", não considerando a idade e a linhagem das matrizes que os produziram. A adoção dessa prática de equalização dos pesos dos ovos é onerosa para o incubatório e não tem sustentação na literatura científica. O objetivo deste trabalho foi verificar a influência da classificação dos ovos no incubatório sobre a uniformidade, o desempenho e o rendimento de abate do frango de corte.

\section{MATERIAL E MÉTODOS}

A composição dos tratamentos foi feita de acordo com Rocha et al. (2008). Foram utilizados ovos incubáveis de matrizes pesadas da linhagem Cobb, produzidos por três lotes de aves com idades de 31,38 e 43 semanas. Os tratamentos, definidos pela classificação dos ovos pela categoria de peso ou pela idade das matrizes, foram: A - ovos com pesos entre $66 \mathrm{e}$ $72 \mathrm{~g}, 33,3 \%$ deles originados de matrizes com diferentes idades; B - ovos com pesos entre $58 \mathrm{e}$ $65 \mathrm{~g}, 33,3 \%$ deles originados de matrizes com diferentes idades; C - ovos com pesos entre $52 \mathrm{e}$ $57 \mathrm{~g}, 33,3 \%$ deles originados de matrizes com diferentes idades; D - ovos com pesos entre $52 \mathrm{e}$ $72 \mathrm{~g}$, produzidos por matrizes com 31 semanas; E - ovos com pesos entre 52 e $72 \mathrm{~g}$, produzidos por matrizes com 38 semanas; e F - ovos com pesos entre 52 e $72 \mathrm{~g}$, produzidos por matrizes com 43 semanas. Os tratamentos A, B e C simularam a classificação que utiliza o peso dos ovos como único critério. Estes foram constituídos igualmente pelas três idades de matrizes para evitar que o efeito de uma idade de matriz influenciasse as respostas obtidas. Os tratamentos D, E e F representaram a classificação mais adequada dos ovos.

Logo após a eclosão, foram coletados ao acaso 1680 pintos, sendo 140 machos e 140 fêmeas de cada um dos seis tratamentos. Os pintos foram alojados em galpão experimental convencional dividido em boxes de $3 \mathrm{~m}^{2}$, onde foram colocadas 
35 aves $\left(11,7\right.$ aves $\left./ \mathrm{m}^{2}\right)$. O manejo dos frangos de um a 44 dias foi semelhante ao utilizado nas criações comerciais, com água e ração oferecidas à vontade.

Três tipos de ração foram utilizados de acordo com as fases de criação, ou seja: inicial - um a 21 dias de idade -, crescimento - 22 a 39 dias de idade -, e acabamento - 40 a 44 dias de idade. A ração utilizada foi a mesma para todos os tratamentos. Para a formulação das rações, foram considerados os valores nutricionais dos ingredientes estabelecidos nas Tabelas Brasileiras de Exigências Nutricionais de Aves e Suínos (Rostagno et al., 2000). Os níveis nutricionais seguiram as recomendações de Lara et al. (2005).

Todas as aves foram pesadas individualmente antes do alojamento, 20 horas após o nascimento, e aos sete e 44 dias de idade, para determinar a uniformidade do peso corporal representada pelos desvios-padrão. As variáveis de desempenho avaliadas aos 44 dias foram: peso corporal, ganho de peso, consumo de ração, conversão alimentar, calculada sobre o ganho de peso e taxa de viabilidade.

O abate foi realizado quando os frangos estavam com 45 dias de idade. Antes do abate, os frangos, foram submetidos a um jejum de ração de 12 horas (Schettino et al., 2006). Os procedimentos de abate foram os mesmos adotados pelo abatedouro, de acordo com as normas do SIF. Para o abate foram apanhados, ao acaso, oito frangos de cada repetição, individualmente identificados, totalizando 384 aves, portanto 32 frangos por tratamento. $\mathrm{Na}$ avaliação do rendimento de carcaça, carcaça limpa eviscerada com pés, cabeça e pescoço, foi considerado o peso vivo em jejum obtido imediatamente antes do abate. Na avaliação dos cortes, coxa + sobrecoxa, peito, dorso, asas, pés, cabeça + pescoço, o rendimento foi feito em relação ao peso da carcaça eviscerada. A gordura abdominal foi retirada após o resfriamento da carcaça em câmara fria. O percentual de gordura abdominal foi calculado em relação ao peso da carcaça eviscerada.

O delineamento experimental foi inteiramente ao acaso, em esquema fatorial $6 \times 2$ (seis tipos de classificação dos ovos $\mathrm{x}$ dois sexos), constituindo 12 tratamentos. Para as avaliações de uniformidade de peso corporal, cada ave foi considerada uma repetição, sendo 140 repetições por tratamento; as variâncias foram comparadas pelo teste de Fisher. Para a avaliação do desempenho dos frangos, o boxe com 35 aves foi considerado a repetição, sendo quatro repetições por tratamento. Para as avaliações do rendimento de abate, cada frango foi considerado como uma repetição, sendo 32 repetições por tratamento. A correlação de Pearson entre os pesos com um, sete e 44 dias foi avaliada pelos pesos individuais das 280 aves de cada tratamento - machos e fêmeas considerados juntos. As médias dos dados de desempenho e rendimento de abate foram comparadas pelo teste SNK (Sampaio, 2002). As análises estatísticas foram realizadas utilizando-se o programa SAEG versão 9.0 (Sistema..., 2005).

\section{RESULTADOS E DISCUSSÃO}

Os dados de desvio-padrão dos pesos das aves com um, sete e 44 dias de idade são apresentados na Tab. 1.

Tabela 1. Desvio-padrão dos pesos dos frangos com um, sete e 44 dias de idade, segundo o tratamento

\begin{tabular}{ccccccc}
\hline \multirow{2}{*}{ Tratamento } & \multicolumn{2}{c}{ Um dia } & \multicolumn{2}{c}{ Sete dias } & \multicolumn{2}{c}{44 dias } \\
\cline { 2 - 7 } & Macho & Fêmea & Macho & Fêmea & Macho & Fêmea \\
\hline A & $1,8 \mathrm{aA}$ & $2,2 \mathrm{bB}$ & $16,7 \mathrm{bA}$ & $15,0 \mathrm{abA}$ & $208,3 \mathrm{abA}$ & $200,1 \mathrm{abA}$ \\
$\mathrm{B}$ & $1,9 \mathrm{aA}$ & $2,0 \mathrm{abA}$ & $13,1 \mathrm{aA}$ & $15,4 \mathrm{bA}$ & $237,1 \mathrm{bA}$ & $217,3 \mathrm{bA}$ \\
$\mathrm{C}$ & $1,7 \mathrm{aA}$ & $1,7 \mathrm{aA}$ & $13,3 \mathrm{aA}$ & $14,3 \mathrm{abA}$ & $215,4 \mathrm{abB}$ & $176,6 \mathrm{aA}$ \\
$\mathrm{D}$ & $2,6 \mathrm{bA}$ & $3,0 \mathrm{cdB}$ & $16,4 \mathrm{bA}$ & $14,0 \mathrm{abA}$ & $237,2 \mathrm{bA}$ & $219,5 \mathrm{bA}$ \\
$\mathrm{E}$ & $3,1 \mathrm{cA}$ & $3,4 \mathrm{dA}$ & $13,0 \mathrm{aA}$ & $15,5 \mathrm{bB}$ & $230,2 \mathrm{abB}$ & $186,4 \mathrm{abA}$ \\
$\mathrm{F}$ & $2,8 \mathrm{bcA}$ & $2,8 \mathrm{cA}$ & $12,7 \mathrm{aA}$ & $12,7 \mathrm{aA}$ & $194,0 \mathrm{aA}$ & $188,2 \mathrm{abA}$ \\
\hline
\end{tabular}

A: ovos com pesos entre 66 e $72 \mathrm{~g}, 33,3 \%$ originados de matrizes com diferentes idades; B: ovos com pesos entre 58 e $65 \mathrm{~g}$, $33,3 \%$ originados de matrizes com diferentes idades; $\mathrm{C}$ : ovos com pesos entre 52 e $57 \mathrm{~g}$, 33,3\% originados de matrizes com diferentes idades; D: ovos com pesos entre 52 e $72 \mathrm{~g}$, produzidos por matrizes com 31 semanas; E: ovos com pesos entre 52 e $72 \mathrm{~g}$, produzidos por matrizes com 38 semanas; $\mathrm{F}$ : ovos com pesos entre 52 e $72 \mathrm{~g}$, produzidos por matrizes com 43 semanas. Uniformidade calculada a partir da variância, porém os dados são apresentados por meio de desvio-padrão dos pesos Valores seguidos de letras distintas, minúsculas na coluna e maiúsculas na linha diferem entre si pelo teste $\mathrm{F}(\mathrm{P} \leq 0,05)$. 
Com um dia de idade, os pintos provenientes de ovos dos tratamentos A, B e C apresentaram os menores desvios-padrão $(\mathrm{P} \leq 0,05)$, ou seja, melhor uniformidade que os pintos provenientes de ovos dos tratamentos D, E e F. Houve interação sexo versus tratamento, sendo os machos provenientes dos tratamentos A e D mais uniformes do que as fêmeas dos respectivos tratamentos $(\mathrm{P} \leq 0,05)$. Machos e fêmeas dos demais tratamentos apresentaram uniformidade semelhante.

Aos sete dias de idade, os machos dos tratamentos A e D foram menos uniformes que os dos demais tratamentos. As fêmeas do tratamento $\mathrm{F}$ foram mais uniformes que as provenientes dos tratamentos $\mathrm{B}$ e $\mathrm{E}(\mathrm{P} \leq 0,05)$. As dos tratamentos $\mathrm{A}, \mathrm{C}$ e $\mathrm{D}$ apresentaram uniformidade semelhante às dos demais tratamentos. Os sexos interagiram com os tratamentos: fêmeas do tratamento $\mathrm{E}$ apresentaram pior uniformidade que os machos desse tratamento. Nos outros tratamentos, não foram observadas diferenças entre sexos quanto à uniformidade.

Aos 44 dias de idade, os machos do tratamento $\mathrm{F}$ obtiveram uniformidade de peso significativamente melhor que os machos dos tratamentos B e D. Os pesos dos machos dos tratamentos $\mathrm{A}, \mathrm{C}$ e $\mathrm{E}$ apresentaram uniformidade semelhante ao dos demais tratamentos $(\mathrm{P}>0,05)$. As fêmeas do tratamento $\mathrm{C}$ foram mais uniformes que as dos tratamentos $\mathrm{B}$ e $\mathrm{D}$ $(\mathrm{P} \leq 0,05)$. Nas dos tratamentos $\mathrm{A}, \mathrm{E}$ e $\mathrm{F}$, os desvios-padrão de peso foram semelhantes aos das fêmeas dos demais tratamentos $(\mathrm{P}>0,05)$. $\mathrm{O}$ sexo interagiu com os tratamentos: as fêmeas dos tratamentos $\mathrm{C}$ e $\mathrm{E}$ foram mais uniformes que os machos dos respectivos tratamentos $(\mathrm{P} \leq 0,05)$. Nos demais tratamentos, não foram observadas diferenças entre machos e fêmeas quanto à uniformidade.

Nos tratamentos A, B e C, observou-se que os pintos tinham pesos mais uniformes com um dia de idade que os dos tratamentos D, E e F. Este resultado já era esperado, pois o peso do pinto é positivamente correlacionado ao peso do ovo (Sinclair, 1990; Pinchasov, 1991), portanto, quanto mais limitada for a faixa de peso dos ovos antes da incubação, maior será a uniformidade do peso dos pintos nascidos desses ovos. Porém, essa melhor uniformidade não foi mantida aos sete e 44 dias de idade dos frangos. Estes achados demonstram que a uniformidade do lote não depende do peso inicial das aves, conseguida por meio da prática de equalização do peso dos ovos no incubatório. Outros fatores relacionados ao manejo dos frangos de corte, como proporcionar número adequado de bebedouros $\mathrm{e}$ comedouros, manter a densidade populacional apropriada e as condições ideais de ambiência, parecem ser mais importantes para manter os lotes uniformes. Segundo Ferreira et al. (2006), evitar o período prolongado de armazenamento dos ovos também contribui para a melhor uniformidade dos frangos de corte.

As correlações de Pearson entre os pesos com um e sete dias, com um e 44 dias e com sete e 44 dias de idade são apresentadas na Tab. 2.

Tabela 2. Correlação de Pearson entre os pesos com um, sete e 44 dias de idade, segundo o tratamento

\begin{tabular}{cccccc}
\hline Tratamento & Peso & 7 dias & 44 dias & Peso & 44 dias \\
\hline A & 1 dia & - & - & 7 dias & 0,82 \\
B & 1 dia & - & - & 7 dias & 0,83 \\
C & 1 dia & 0,76 & - & 7 dias & 0,77 \\
D & 1 dia & - & - & 7 dias & - \\
E & 1 dia & - & - & 7 dias & 0,79 \\
F & 1 dia & - & - & 7 dias & 0,75
\end{tabular}

A: ovos com pesos entre 66 e 72g, 33,3\% originados de matrizes com diferentes idades; B: ovos com pesos entre 58 e $65 \mathrm{~g}$, $33,3 \%$ originados de matrizes com diferentes idades; C: ovos com pesos entre 52 e $57 \mathrm{~g}, 33,3 \%$ originados de matrizes com diferentes idades; D: ovos com pesos entre $52 \mathrm{e} 72 \mathrm{~g}$, produzidos por matrizes com 31 semanas; E: ovos com pesos entre $52 \mathrm{e}$ $72 \mathrm{~g}$, produzidos por matrizes com 38 semanas; F: ovos com pesos entre $52 \mathrm{e} 72 \mathrm{~g}$, produzidos por matrizes com 43 semanas.

Entre as aves do tratamento C, observou-se correlação alta e positiva entre os pesos com um e sete dias de idade; essa correlação não foi observada entre as aves dos demais tratamentos. Não foi observada correlação entre o peso do pinto com um dia e o peso do frango aos 44 dias de idade em nenhum dos tratamentos. O peso do pinto aos sete dias foi positivamente correlacionado ao peso do frango aos 44 dias em todos os tratamentos, exceto em aves do 
tratamento D. Estes resultados estão de acordo com Baião (2000) e Tona et al. (2004; 2005). A não correlação entre os pesos dos pintos com um e 44 dias de idade em todos os tratamentos demonstrou que o peso inicial das aves não influenciou o peso corporal delas aos 44 dias de idade.

No tratamento $\mathrm{D}$, a não correlação entre peso nas idades de sete e 44 dias pode ser explicado pelo fato de os pintos terem sido os mais leves aos sete dias $(\mathrm{P} \leq 0,05)$ quando comparados com os dos demais tratamentos. Aos 44 dias, os pesos foram semelhantes para todos os tratamentos, exceto para A (Tab. 3).

Os resultados do peso corporal, do consumo de ração, da conversão alimentar e da viabilidade dos pintos de um a 44 dias são apresentados na Tab. 3.

Tabela 3. Desempenho dos frangos de um a 44 dias de idade, segundo o tratamento

\begin{tabular}{|c|c|c|c|c|c|c|c|}
\hline \multirow{2}{*}{ Sexo } & \multicolumn{7}{|c|}{ Tratamento } \\
\hline & A & B & $\mathrm{C}$ & $\mathrm{D}$ & $\mathrm{E}$ & $\mathrm{F}$ & Média \\
\hline \multicolumn{8}{|c|}{ Peso corporal $(\mathrm{g})$} \\
\hline Fêmea & 2570 & 2475 & 2475 & 2469 & 2515 & 2494 & $2500 \mathrm{~b}$ \\
\hline Macho & 3128 & 3024 & 3010 & 2988 & 3031 & 3088 & $3045 a$ \\
\hline Média & 2849A & 2749B & 2743B & 2729B & 2773B & $2791 \mathrm{AB}$ & \\
\hline $\mathrm{CV}$ & $2,07 \%$ & & & & & & \\
\hline \multicolumn{8}{|c|}{ Ganho de peso (g) } \\
\hline Fêmea & 2524 & 2435 & 2439 & 2432 & 2475 & 2451 & $2459 b$ \\
\hline Macho & 3083 & 2983 & 2974 & 2951 & 2991 & 3045 & $3004 a$ \\
\hline Média & $2804 \mathrm{~A}$ & 2709B & 2706B & 2691B & 2733B & $2748 \mathrm{AB}$ & \\
\hline $\mathrm{CV}$ & $2,11 \%$ & & & & & & \\
\hline \multicolumn{8}{|c|}{ Consumo de ração $(\mathrm{g})$} \\
\hline Fêmea & 4383 & 4243 & 4228 & 4249 & 4256 & 4305 & $4277 b$ \\
\hline Macho & 5068 & 5069 & 4955 & 4852 & 5022 & 5108 & $5012 \mathrm{a}$ \\
\hline Média & $4725 \mathrm{~A}$ & 4656ABC & $4591 B C$ & $4550 \mathrm{C}$ & 4639ABC & $4706 \mathrm{AB}$ & \\
\hline $\mathrm{CV}$ & $1,90 \%$ & & & & & & \\
\hline \multicolumn{8}{|c|}{ Conversão alimentar $(\mathrm{kg} / \mathrm{kg})$} \\
\hline Fêmea & 1,74 & 1,75 & 1,73 & 1,75 & 1,72 & 1,76 & $1,74 b$ \\
\hline Macho & 1,64 & 1,70 & 1,67 & 1,64 & 1,68 & 1,68 & $1,67 \mathrm{a}$ \\
\hline Média & $1,69 \mathrm{~A}$ & $1,72 \mathrm{~A}$ & $1,70 \mathrm{~A}$ & $1,70 \mathrm{~A}$ & $1,70 \mathrm{~A}$ & $1,72 \mathrm{~A}$ & \\
\hline $\mathrm{CV}$ & $1,98 \%$ & & & & & & \\
\hline \multicolumn{8}{|c|}{ Viabilidade $(\%)$} \\
\hline Fêmea & 97,9 & 95,1 & 98,6 & 97,1 & 96,4 & 97,1 & $97,0 \mathrm{a}$ \\
\hline Macho & 90,7 & 94,2 & 92,1 & 92,9 & 90,7 & 95,1 & $92,6 b$ \\
\hline Média & $94,3 \mathrm{~A}$ & $94,6 \mathrm{~A}$ & $95,4 \mathrm{~A}$ & $95,0 \mathrm{~A}$ & $93,6 \mathrm{~A}$ & $96,1 \mathrm{~A}$ & \\
\hline $\mathrm{CV}$ & $3,87 \%$ & & & & & & \\
\hline
\end{tabular}

A: ovos com pesos entre 66 e $72 \mathrm{~g}, 33,3 \%$ originados de matrizes com diferentes idades; B: ovos com pesos entre 58 e $65 \mathrm{~g}$, $33,3 \%$ originados de matrizes com diferentes idades; C: ovos com pesos entre 52 e $57 \mathrm{~g}$, 33,3\% originados de matrizes com diferentes idades; D: ovos com pesos entre 52 e $72 \mathrm{~g}$, produzidos por matrizes com 31 semanas; E: ovos com pesos entre 52 e $72 \mathrm{~g}$, produzidos por matrizes com 38 semanas; F: ovos com pesos entre $52 \mathrm{e} 72 \mathrm{~g}$, produzidos por matrizes com 43 semanas. Valores seguidos de letras distintas, minúsculas na coluna e maiúsculas na linha, diferem entre si pelo teste $\mathrm{SNK}(\mathrm{P} \leq 0,05)$.

Não foi observada interação tratamento versus sexo em nenhuma das variáveis de desempenho nessa idade. Independente do tratamento, o sexo influenciou todas as variáveis de desempenho, portanto os machos apresentaram maiores peso corporal, ganho de peso e consumo de ração, melhor conversão alimentar e pior viabilidade quando comparados às fêmeas aos 44 dias de idade $(\mathrm{P} \leq 0,05)$.
Independente do sexo, os frangos originados do tratamento A apresentaram maior peso corporal e ganho de peso que os provenientes dos tratamentos $\mathrm{B}, \mathrm{C}, \mathrm{D}$ e $\mathrm{E}(\mathrm{P} \leq 0,05)$. Os oriundos do tratamento $\mathrm{F}$ apresentaram peso corporal $\mathrm{e}$ ganho de peso semelhantes a todos os tratamentos. Estes resultados estão de acordo com os observados por Wyatt et al. (1985), Wilson (1991), Vieira e Moran Jr. (1998), Tona et al. (2004) e Joseph e Moran Jr. (2005). Isto 


\section{Rocha et al.}

explica porque o peso do pinto com um dia e o peso do frango aos 44 dias de idade não foram correlacionados (Tab. 2), pois o fator que influenciou o peso corporal aos 44 dias de idade foi a capacidade de ganho de peso das aves, independente de seu peso inicial.

Os frangos do tratamento $\mathrm{D}$ consumiram menos ração que os dos tratamentos $\mathrm{A}$ e $\mathrm{F}(\mathrm{P} \leq 0,05)$.

Independente do sexo, não foram encontradas diferenças na conversão alimentar e na viabilidade entre os tratamentos. Os resultados da conversão alimentar estão de acordo com os de Delanezi et al. (2005), e os da viabilidade com os de Tona et al. (2004) e Joseph e Moran Jr. (2005).

Os dados de rendimento de carcaça, de coxa + sobrecoxa e de peito, obtidos no abate aos 45 dias de idade, são apresentados na Tab. 4.

Tabela 4. Rendimento (\%) de carcaça, de coxa + sobrecoxa e de peito dos frangos, machos e fêmeas, segundo os tratamentos

\begin{tabular}{ccc}
\hline Tratamento & \multicolumn{2}{c}{ Sexo } \\
\cline { 2 - 3 } & Fendimento de carcaça (\%) & Macho \\
\hline A & $86,7 \mathrm{aB}$ & $87,8 \mathrm{aA}$ \\
B & $85,2 \mathrm{aB}$ & $88,1 \mathrm{aA}$ \\
C & $86,0 \mathrm{aA}$ & $87,0 \mathrm{aA}$ \\
D & $86,2 \mathrm{aB}$ & $88,2 \mathrm{aA}$ \\
E & $87,7 \mathrm{aA}$ & $87,7 \mathrm{aA}$ \\
F & $86,7 \mathrm{aB}$ & $88,0 \mathrm{aA}$ \\
CV & $2,59 \%$ & \\
\hline A & $26,6 \mathrm{aB}$ & $27,6 \mathrm{aA}$ \\
B & $26,7 \mathrm{aB}$ & $28,0 \mathrm{aA}$ \\
C & $26,6 \mathrm{aB}$ & $27,7 \mathrm{aA}$ \\
D & $27,0 \mathrm{aA}$ & $26,8 \mathrm{aA}$ \\
E & $26,2 \mathrm{aB}$ & $27,7 \mathrm{aA}$ \\
F & $26,0 \mathrm{aB}$ & $27,6 \mathrm{aA}$ \\
CV & $4,67 \%$ & \\
\hline A & Rendimento de coxa & $34,9 \mathrm{aB}$ \\
B & $37,6 \mathrm{aA}$ & $34,5 \mathrm{aB}$ \\
C & $37,5 \mathrm{aA}$ & $35,2 \mathrm{aB}$ \\
D & $36,6 \mathrm{aA}$ & $35,8 \mathrm{aA}$ \\
E & $36,3 \mathrm{aA}$ & $35,0 \mathrm{aB}$ \\
CV & $37,0 \mathrm{aA}$ & $35,0 \mathrm{aB}$ \\
\hline
\end{tabular}

A: ovos com pesos entre 66 e $72 \mathrm{~g}, 33,3 \%$ originados de matrizes com diferentes idades; B: ovos com pesos entre 58 e $65 \mathrm{~g}$, $33,3 \%$ originados de matrizes com diferentes idades; $\mathrm{C}$ : ovos com pesos entre 52 e $57 \mathrm{~g}$, 33,3\% originados de matrizes com diferentes idades; D: ovos com pesos entre 52 e $72 \mathrm{~g}$, produzidos por matrizes com 31 semanas; E: ovos com pesos entre 52 e $72 \mathrm{~g}$, produzidos por matrizes com 38 semanas; $\mathrm{F}$ : ovos com pesos entre 52 e $72 \mathrm{~g}$, produzidos por matrizes com 43 semanas. Valores seguidos de letras distintas, minúsculas na coluna e maiúsculas na linha diferem entre si pelo teste $\mathrm{SNK}(\mathrm{P} \leq 0,05)$.

Independente do sexo, não houve diferenças no rendimento de carcaça entre os tratamentos. Estes resultados estão de acordo com os observados por Vieira e Moran Jr. (1998) e Joseph e Moran Jr. (2005). Sexo interagiu com tratamentos, isto é, o rendimento dos machos foi maior que o das fêmeas somente nos tratamentos $\mathrm{A}, \mathrm{B}, \mathrm{D}$ e $\mathrm{F}(\mathrm{P} \leq 0,05)$. Nos tratamentos $\mathrm{C}$ e $\mathrm{E}, \mathrm{o}$ rendimento de carcaça foi semelhante entre fêmeas e machos.

Houve interação sexo versus tratamento no estudo de rendimento de coxa + sobrecoxa. No tratamento D, as fêmeas apresentaram rendimento igual ao dos machos. Nos demais tratamentos, a fêmea apresentou rendimento de 
coxa + sobrecoxa inferior $(\mathrm{P} \leq 0,05)$ ao dos machos. Independente do sexo, não houve diferença estatística entre os tratamentos para essa variável. Este resultado coincide com os de Vieira e Moran Jr. (1998) e Delanezi et al. (2005).

Os resultados de rendimento de peito mostraram que houve interação sexo versus tratamento. $\mathrm{O}$ rendimento de peito dos machos foi menor que o das fêmeas em todos os tratamentos, exceto no tratamento D. Independente do sexo, não foi observada diferença significativa no rendimento de peito entre os tratamentos. Esta observação está de acordo com Vieira e Moran Jr. (1998).

Não houve interação sexo versus tratamento para as variáveis rendimento de asas, rendimento de dorso, rendimento de cabeça + pescoço, rendimento de pés e percentual de gordura abdominal. Independente do tratamento, quando comparadas aos machos, as fêmeas apresentaram melhores rendimentos de asa e de dorso, piores rendimentos de cabeça + pescoço e de pés, e maior percentual de gordura abdominal $(\mathrm{P} \leq 0,05)$. Independente do sexo, não foi observada diferença estatística entre os tratamentos para as variáveis citadas.

\section{CONCLUSÕES}

A prática de classificação dos ovos, considerando somente a categoria de peso, é ineficaz para garantir a uniformidade dos frangos de corte ao longo do período de criação. O desempenho e o rendimento de abate independem da classificação dos ovos no incubatório.

\section{REFERÊNCIAS BIBLIOGRÁFICAS}

BAIÃO, N.C. O peso no abate. Avimig, n.1, p.12-14, 2000.

ELANEZI, J.A.; MENDES, A.A.; GARCIA, E.A. et al. Efeito da idade da matriz sobre o desempenho e rendimento de carcaça de frangos de corte. Arq. Bras. Med. Vet. Zootec., v.57, p.250-260, 2005.

FERREIRA, F.C.; LARA, L.J.C.; BAIÃO, N.C. et al. Influência da idade da matriz e do período de armazenamento dos ovos sobre a uniformidade dos pintos aos 11 dias de idade. Rev. Bras. Cien. Avic., v.8, supl., p.17, 2006.
JOSEPH, N.S.; MORAN Jr., E.T. Effect of flock age and postemergent holdin in the hatcher on broiler live performance and further-processing yeld. J. Appl. Poult. Res., v.14, p.512-520, 2005.

LARA, L.J.C.; BAIÃO, N.C.; AGUILAR, C.A.L. et al. Efeito de fontes lipídicas sobre o desempenho de frangos de corte. Arq. Bras. Med. Vet. Zootec., v.57, p.792-798, 2005.

PINCHASOV, Y. Relationship between the weight of hatching eggs and subsequent early performance of broiler chicks. Poult. Sci., v.32, p.109-115, 1991.

ROSTAGNO, H.S.; ALBINO, L.F.T.; DONZELE, J.L. et al. Tabelas brasileiras para aves e suinos composição de alimentos e exigências nutricionais. Viçosa: UFV, 2000. 141p.

ROCHA, J.S.R.; LARA, L.J.C.; BAIÃO, N.C. et al. Efeito da classificação dos ovos sobre o rendimento de incubação e os pesos do pinto e do saco vitelino. Arq. Bras. Med. Vet. Zootec., v.60, p.979-986, 2008.

SAMPAIO, I.B.M. Estatística aplicada à experimentação animal. 2.ed. Belo Horizonte: FEPMVZ, 2002. 265p.

SCHETTINO, D.N., CANÇADO, S.V.; BAIÃO, N.C. et al. Efeito do período de jejum pré-abate sobre o rendimento de carcaça de frango de corte. Arq. Bras. Med. Vet. Zootec., v.58, p.918-924, 2006.

SINCLAIR, R.W.; ROBINSON, F.E.; HARDIN, R.T. The effects of parent age and posthatch treatment on broiler performance. Poult. Sci., v.69, p.526-534, 1990.

SISTEMA de análises estatísticas. Versão 9.0 SAEG. Viçosa: UFV, 2005.

TONA, K.; BRUGGEMAN, V.; ONAGBESAN, O. et al. Day-old chick quality: relationship to hatching egg quality, adequate incubation practice and prediction of broiler performace. Avian Poult. Biol. Rev., v.16, p.109-119, 2005.

TONA, K.; ONAGBESAN, O.; DE KETELAERE, et al. Effects of age of broiler breeders and egg storage on egg quality, hatchability, chick quality, chick weight, and chick posthatch growth to forty-two days. J. Appl. Poult. Res., v.13, p.10-18, 2004.

VIEIRA, S.L.; MORAN Jr., E.T. Broiler yields using chicks from egg weight extremes and diverse strains. J. Appl. Poult. Res., v.7, p.339-346, 1998.

WILSON, H.R. Interrelationships of egg size, chick size, posthatching growth and hatchability. World's Poult. Sci. J., v.47, p.5-20, 1991.

WYATT, C.L.; WEAVER, J.R.; BEANE, W.L. Influence of egg size, eggshell quality, and posthatch holding time on broiler performance. Poult. Sci., v.64, p.2049-2055, 1985. 